\title{
Seismic waves in fractured porous media
}

\author{
J.-L. Auriault* and P. Royer* \\ *3S-R - Laboratoire sols, solides, structures - risques, Grenoble
}

\section{INTRODUCTION}

A fractured porous medium is a dual-porosity medium, i.e., it consists of two interacting porous systems whose permeabilities are very different. The purpose of this paper is to mathematically model seismic wave propagation in saturated fractured porous media when the wavelength is large compared to the fracture characteristic length.

The acoustics of porous saturated media are of first importance in numerous engineering areas, e.g., seismic exploration, property testings in paper manufacturing, designing antinoise devices. When modeling transport processes in porous media, we can distinguish two kind of approaches (1) phenomenological approaches, i.e., macroscopic approaches, and (2) homogenization methods by which the macroscopic equations are derived by starting with the detailed microscale physical description. The acoustics of single-porosity media, i.e., porous media with a single pore-size characteristic length, was pioneered by Biot, who derived his model on the basis of phenomenological reasoning (Biot, 1956). In Biot's theory, the porous matrix is assumed to be elastic, and the fluid is vis-cous and Newtonian. Biot's model is a two-phase model: the porous saturated medium is described by two macroscopic displacement fields, $\mathbf{u}_{s}$ for the porous matrix and $\mathbf{u}_{f}$ for the fluid. In describing the relative fluid-solid movement, the solid displacement is effectively constant at the microscale, which shows that, at this scale, its movement is a rigid translation. As for the local fluid displacement, it depends upon the position within the pore. Thus, $\mathbf{u}_{f}$ stands for its average throughout an aver-aging volume. For a slightly compressible or an incompressible fluid at constant angular frequency, $\omega$, Biot's model reads

$$
\begin{aligned}
\frac{\partial \Sigma_{i j}^{T}}{\partial x_{j}} & =-(1-\Phi) \omega^{2} \rho_{s} u_{s i}-\omega^{2} \rho_{f} u_{f i}, \\
\Sigma_{i j}^{T} & =C_{i j k l} E_{k l}\left(\mathbf{u}_{s}\right)-\Gamma_{i j} p
\end{aligned}
$$

$$
\begin{aligned}
\frac{\partial\left(i \omega u_{f i}-i \omega \Phi u_{s i}\right)}{\partial x_{i}} & =-\Pi_{i j} i \omega E_{i j}\left(\mathbf{u}_{s}\right)-b i \omega p \\
i \omega u_{f i}-i \omega \Phi u_{s i} & =-K_{i j}\left(\frac{\partial p}{\partial x_{j}}-\omega^{2} \rho_{f} u_{s j}\right)
\end{aligned}
$$

where $\boldsymbol{\Sigma}^{T}$ is the total stress; $\rho_{s}$ and $\rho_{f}$ are the solid and the fluid densities, respectively; $\mathbf{E}$ is deformation; $\Phi$ is porosity; $P$ is the pressure; $C_{i j k l}, \Gamma_{i j}=\Pi_{i j}$, and $b$ are elastic coefficients; and $K_{i j}$ is the acoustic permeability, which is complex valued and frequency dependent. An important feature of Biot's model is the symmetry $\Gamma_{i j}=\Pi_{i j}$. Equation (4) represents the acoustic filtration law. Biot's model has been derived by homogenization in Levy (1979), Auriault (1980), and Burridge and Keller (1981). Homogenization has also proven that Biot's model [equations (1)-(4)] is not the only mathematical model for describing the acoustics of elastic porous media saturated by a viscous Newtonian fluid (Auriault 1991a)

A dual-porosity medium consists of two interacting porous systems of distinctly different fluid transport properties, as is the case in a fractured porous medium: one porous structure is associated with the fractures, and the second is associated with the porous matrix. The concept of dual porosity was introduced by Barenblatt et al. (1960) to investigate fluid flow in naturally fractured porous media. A few attempts have been made, using phenomenological reasoning, to generalize Biot's theory for wave propagation in saturated fractured media (Wilson and Aifantis, 1984; Beskos, 1989; Berryman and Wang, 2000). By homogenization, Auriault and Boutin (1994) derived four distinct macroscopic models. The objective of our paper is to summarize this work on deriving the equations that control wave propagation in saturated fractured media.

Fractured porous media are characterized by the existence of three separate scales of distinctly different characteristic lengths: the pore scale, the fracture scale, and the macroscopic scale (see Figure 1). When investigating wave propagation, 
the macroscopic characteristic length to be considered is the wavelength $\lambda$. Let's denote by $\ell$ and $\ell^{\prime}$ the pore-scale and the fracture-scale characteristic lengths, respectively. We therefore have

$$
\ell \ll \ell^{\prime} \ll \lambda .
$$

A three-scale homogenization method is therefore used. The only hypotheses are in the laws used to describe the physics at the pore and fracture scales. The porous, fractured matrix is assumed to be elastic, and the fluid within the pores and fractures is viscous Newtonian and incompressible or slightly compressible. The three-scale homogenization method consists of homogenizing the pore-scale description to derive the porous matrix behavior and homogenizing the fracture-scale description to obtain the macroscopic equations. The macroscopic behavior depends strongly upon the relative order of magnitude of the scale ratios $\ell / \ell^{\prime}$ and $\ell^{\prime} / \lambda$.

The analysis below is restricted to the case of a fractured porous medium subject to a two-phase Biot's regime in the fractures for which four distinct macroscopic two-phase models are obtained. In effect, single-phase regimes occur when there is no local flow and are therefore of lower interest.

The next section is devoted to a brief, general presentation of the homogenization method. A following section considers the homogenized models for acoustics in single-porosity media. The last section presents the models obtained for describing wave propagation in dual-porosity media. Attention is focused on the results obtained by the homogenization process. For details related to the derivation of the models, the reader is referred, when required, to other references.

\section{HOMOGENIZATION METHOD}

The essence of homogenization techniques is to determine an equivalent macroscopic behavior by upscaling the

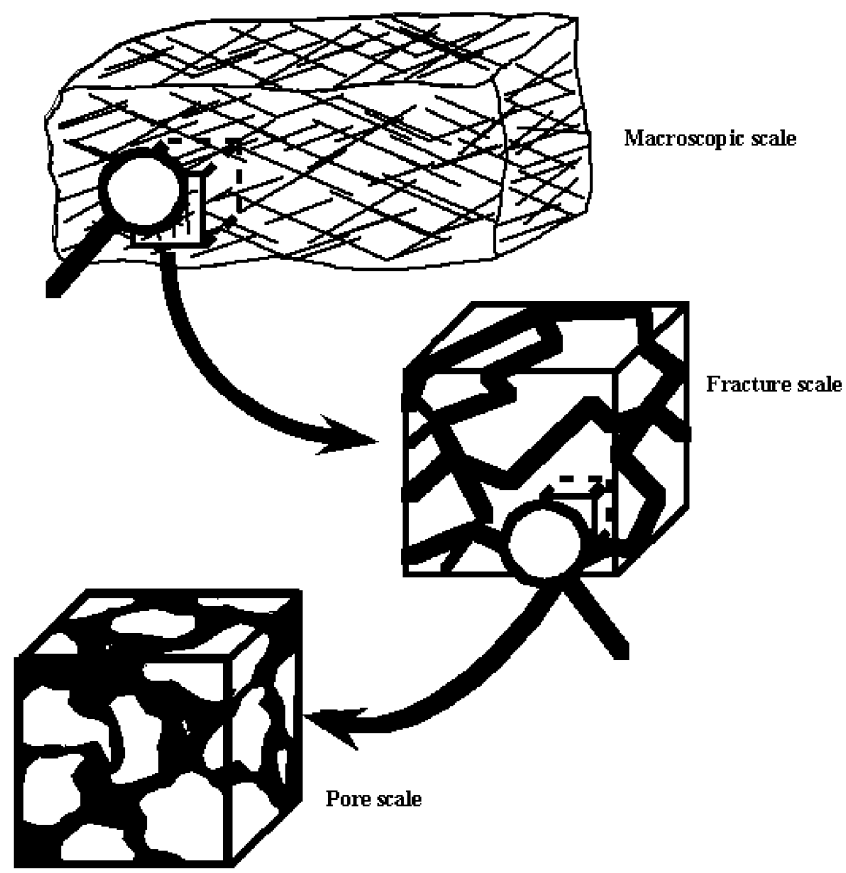

FIG. 1. The three scales of a fractured porous medium. local description. The fundamental assumption behind any homogenization method is that the scales must be separate. For a two-scale medium, this condition is expressed as $\ell \ll L$, where $\ell$ and $L$ are the characteristic lengths at the heterogeneity scale and the macroscopic scale, respectively. This definition conjures up a purely geometric separation of scales, but this fundamental condition must also be satisfied by the physical process considered. For example, for wave propagation in a heterogeneous medium, the heterogeneity characteristic length $\ell$ must also be small compared to the wavelength.

We use the homogenization method for periodic structures (also called method of multiple scales) introduced by Keller (1977), Bensoussan et al. (1978), and Sanchez-Palencia (1980). When dealing with a two-scale medium, the key parameter of the method is the small parameter

$$
\varepsilon=\frac{\ell}{L} \ll 1
$$

Depending on the problem under consideration, $L$ is either geometric (i.e., the sample size) or related to excitation (e.g., wavelength). With this homogenization method, the medium is also assumed to be periodic. This assumption is actually not a restriction; it allows derivation of the macroscopic model without any assumption on the form of the macroscopic equations. In this study, we use the formulation of the method suggested in Auriault (1991b), with which the problem is tackled in a more physical manner. This formulation uses the dimensionless numbers that arise from the local description. These dimensionless numbers must be estimated with respect to the scale ratio $\varepsilon$. Using the two characteristic lengths, two dimensionless space variables are defined:

$$
\mathbf{y}=\frac{\mathbf{x}}{\ell}, \quad \mathbf{y}^{\prime}=\frac{\mathbf{x}}{L},
$$

where $\mathbf{x}$ is the physical space variable. If the condition of separation of scales is satisfied, then $\mathbf{y}$ and $\mathbf{y}^{\prime}$ appear as two independent space variables: $\mathbf{y}$ is the microscopic variable and describes the heterogeneity scale, whereas $\mathbf{y}^{\prime}$ is the macroscopic variable. As a consequence, the physical variables of the problem, $p$ and $\mathbf{u}$, are a priori functions of $\mathbf{y}$ and $\mathbf{y}^{\prime}$ :

$$
p=p\left(\mathbf{y}, \mathbf{y}^{\prime}\right), \quad \mathbf{u}=\mathbf{u}\left(\mathbf{y}, \mathbf{y}^{\prime}\right) .
$$

Such a separation into $\mathbf{y}$ and $\mathbf{y}^{\prime}$ dependence is fully justified in an asymptotic sense. The homogenization method of multiple scales is based on the fundamental premise that if the scales are well separated, then all physical variables can be looked for in the form of asymptotic expansions in powers of $\varepsilon$ :

$$
\begin{aligned}
& p=p^{0}\left(\mathbf{y}, \mathbf{y}^{\prime}\right)+\varepsilon p^{1}\left(\mathbf{y}, \mathbf{y}^{\prime}\right)+\cdots, \\
& \mathbf{u}=\mathbf{u}^{0}\left(\mathbf{y}, \mathbf{y}^{\prime}\right)+\varepsilon \mathbf{u}^{1}\left(\mathbf{y}, \mathbf{y}^{\prime}\right)+\cdots,
\end{aligned}
$$

in which the functions $p^{i}$ and $\mathbf{u}^{i}$ are $\mathbf{y}$-periodic.

The method consists of incorporating expansions (8) and (9) in the dimensionless form of the local description. Solving the boundary-value problems arising at the successive orders of $\varepsilon$ leads to the macroscopic description.

When considering a dual-porosity medium, this methodology must be extended to three-scale problems. Thus, three dimensionless space variables must be defined and two scale ratios be considered. This three-scale homogenization method, 
established by Auriault and Boutin $(1992,1993)$ and has been applied successfully to various dual-porosity problems.

\section{SINGLE-POROSITY MEDIA}

Let's denote by $\Omega_{s}$ and $\Omega_{f}$ the solid and fluid parts of the period, respectively, and let $\Gamma$ be the pore surface. The local, i.e., pore-scale, description is given by

$$
\begin{aligned}
\frac{\partial \Sigma_{\alpha i j}}{\partial x_{j}} & =-\omega^{2} \rho_{\alpha} u_{\alpha i}, \quad \text { in } \Omega_{\alpha}, \alpha=s, f, \\
\Sigma_{s i j} & =A_{i j k l} E_{k l}\left(\mathbf{u}_{s}\right), \\
\Sigma_{f i j} & =-p I_{i j}+2 \mu E_{i j}\left(i \omega \mathbf{u}_{f}\right), \\
\frac{\partial u_{f i}}{\partial x_{i}} & =\frac{-p}{K_{w}}, \quad \text { in } \Omega_{f}, \\
\Sigma_{s i j} n_{j} & =\Sigma_{f i j} n_{j}, \\
u_{s i} & =u_{f i} \quad \text { on } \Gamma,
\end{aligned}
$$

where $\rho_{\alpha}(\alpha=s, f)$ is the density, $I$ is the identity tensor, $A$ is the elastic rigidity of the solid, $\mu$ is the viscosity of the fluid, and $K_{w}$ is the rigidity of the fluid. Equation (10) is the momentumbalance equation; the behaviors of the solid and the liquid are given by equation (11). Equation (12) is the mass-balance equation for the fluid and is valid for an incompressible or a slightly compressible fluid. Equations (13) and (14) are the boundary conditions on $\Gamma$ that express the continuity of normal stresses and displacements, respectively.

It turns out that the macroscopic behavior is fully conditioned by the order of magnitude of the following dimensionless number (Auriault, 1991b),

$$
R=\frac{|\mathbf{C}|}{\omega \mu},
$$

where $|\mathbf{C}|$ is an a priori estimate of the porous matrix elastic modulus. The value $R$ is linked to boundary condition (13) and characterizes the local fluid-solid movement. Four macroscopic models are obtained that correspond to four orders of magnitude for the property ratio $R$. The model classification below is made with decreasing orders of $R$ which, assuming fixed values for $|\mathbf{C}|$ and $\omega$, corresponds to increasing values for $\mu$.

The order of magnitude with respect to $\varepsilon$ is defined by $R=O\left(\varepsilon^{p}\right), p$ integer, if $\varepsilon^{p+1} \ll R \ll \varepsilon^{p-1}$.

Model $\boldsymbol{A}: \boldsymbol{R} \geq \boldsymbol{O}\left(\varepsilon^{-\mathbf{3}}\right)$. - At the first order of approximation, the saturated porous medium behaves like an empty elastic porous medium. The quality factor $Q^{-1}$ is defined as the maximum value, with respect to $\omega$, of the ratio of the dissipation energy during a cycle to the maximum elastic energy. In the present case there is no viscous effect: the quality factor is therefore negligible and is estimated as $Q^{-1}=O(\varepsilon)$.

Model $\boldsymbol{B}: \boldsymbol{R}=\boldsymbol{O}\left(\varepsilon^{-\mathbf{2}}\right)$.- There is movement of the fluid relative to the skeleton. The macroscopic behavior is given by Biot's model [equations (1)-(4)]. The quality factor is $Q^{-1}=O(1)$.

Model $\boldsymbol{C}: \boldsymbol{R}=\boldsymbol{O}\left(\varepsilon^{\mathbf{- 1}}\right)$.- The macroscopic model describes single-phase elastic behavior but differs from model A. In effect, in contrast to model $A$, which describes the behavior of an empty matrix, the present model describes the behavior of a impermeable porous matrix saturated by a fluid. The fluid has an influence on the elastic properties of the material. The quality factor is $Q^{-1}=O(\varepsilon)$.

Model $\boldsymbol{D}: \boldsymbol{R}=\boldsymbol{O ( 1 )}$.- There is a very strong influence from the fluid properties. The macroscopic model represents a single-phase viscoelastic behavior. The quality factor is $Q^{-1}=O(1)$.

The four models are summarized in Figure 2.

\section{DUAL-POROSITY MEDIA}

When looking for the macroscopic behavior of wave propagation in fractured porous media, three scales (pore, fracture, and macroscopic) and, consequently, two scale ratios must be considered:

$$
\begin{aligned}
& \alpha=\frac{\ell}{\ell^{\prime}} \ll 1 \quad \text { pore/fracture scale ratio, } \\
& \beta=\frac{\ell^{\prime}}{\lambda} \ll 1 \quad \text { fracture/wavelength scale ratio. }
\end{aligned}
$$

The macroscopic behavior depends upon the orders of magnitude of the property ratios:

$$
\begin{aligned}
& R_{p}=\frac{|C|}{\omega \mu} \quad \text { pore-scale property ratio, } \\
& R_{f}=\frac{|D|}{\omega \mu} \quad \text { fracture-scale property ratio, }
\end{aligned}
$$

where $|C|$ and $|D|$ are a priori estimates of the elastic moduli of the porous matrix (fracture scale) and the fractured matrix (macroscopic scale), respectively. More precisely, the macroscopic behavior is conditioned by the order of $R_{p}$ with respect to $\alpha$, which determines the regime in the porous matrix, and by the order of $R_{f}$ with respect to $\beta$, which characterizes the type of macroscopic model. The results obtained in the singleporosity case indicate that a two-phase model (B) corresponds to $R=O\left(\varepsilon^{-2}\right)$. Thus, to obtain two-phase dual-porosity models (which effectively correspond to an extension of Biot's theory to fractured porous media), we restrict the analysis to

$$
R_{f}=O\left(\beta^{-2}\right),
$$

for which the quality factor is $Q_{f}=O(1)$. Two-phase models are those of greatest interest for geophysical applications since in the corresponding situations there exists a movement of the fluid relatively to the matrix. As a consequence, the signature of the fluid flow is contained in these models. Once $R_{f}$ has been fixed, the behavior remains conditioned by the order of $R_{p}$ with respect to $\alpha$ :

$$
R_{p}=O\left(\alpha^{p}\right)
$$

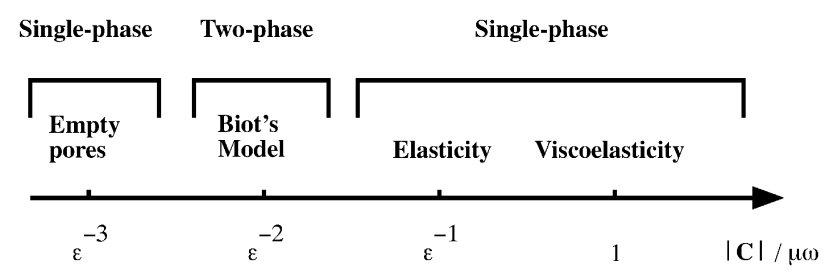

FIG. 2. The four behaviors of a single-porosity medium. 
This will determine the regime in the porous matrix. We generally have $D=O(C)$; hence, $R_{p}$ and $R_{f}$ are the same order:

$$
R_{p}=O\left(\alpha^{p}\right)=O\left(R_{f}\right)=O\left(\beta^{-2}\right) .
$$

Therefore, the macroscopic behavior is conditioned by the relative order of magnitude of both scale ratios:

$$
\alpha=O\left(\beta^{q}\right), q \text { integer. }
$$

Four macroscopic models are obtained that correspond to that of Auriault and Boutin (1994):

$\alpha=\beta^{1 / 2} ; \quad \alpha=O(\beta) ; \alpha=O\left(\beta^{2}\right) ; \alpha=O\left(\beta^{n}\right), \quad n>4$.

When $\alpha=\beta^{1 / 2}$, we get $R_{p}=O\left(\alpha^{-4}\right)$ which, according to the single-porosity classification, corresponds to a type A regime in the porous matrix. This dual-porosity model will therefore be type B-A. Thus, we get the following classification for the four models:

$$
\begin{array}{lrl}
\bullet \alpha=\beta^{1 / 2}: & R_{p}=O\left(\alpha^{-4}\right) \Rightarrow \operatorname{model} \mathrm{B}-\mathrm{A}, \\
\bullet \alpha=O(\beta): & R_{p}=O\left(\alpha^{-2}\right) \Rightarrow \operatorname{model} \mathrm{B}-\mathrm{B}, \\
\bullet \alpha=O\left(\beta^{2}\right): & R_{p}=O\left(\alpha^{-1}\right) \Rightarrow \operatorname{model} \mathrm{B}-\mathrm{C}, \\
\bullet \alpha=O\left(\beta^{n}\right), n>4: & R_{p}=O\left(\alpha^{0}\right) \Rightarrow \text { model B-D. }
\end{array}
$$

\section{Model $B-A$ : Low pore-fracture separation of scales}

Model $B-A$ is defined by $\ell / \ell^{\prime}=O\left(\sqrt{\ell^{\prime} / \lambda}\right)$ and is given by

$$
\begin{aligned}
\frac{\partial \Sigma_{i j}^{T}}{\partial x_{j}}= & -\left[\left(1-\Phi_{p}\right) \rho_{s}+\Phi_{p} \rho_{f}\right]\left(1-\Phi_{f}\right) \\
& \times \omega^{2} u_{s i}-\omega^{2} \rho_{f} u_{f i} \\
\Sigma_{i j}^{T}= & D_{i j k l} E_{x k l}\left(\mathbf{u}_{s}\right)-\Gamma_{i j} p \\
\frac{\partial\left(i \omega u_{f i}-i \omega \Phi_{f} u_{s i}\right)}{\partial x_{i}}= & -\Pi_{i j} i \omega E_{x i j}\left(\mathbf{u}_{s}\right)-b i \omega p \\
i \omega u_{f i}-i \omega \Phi_{f} u_{s i}= & -K_{f i j}\left(\frac{\partial p}{\partial x_{j}}-\omega^{2} \rho_{f} u_{s j}\right)
\end{aligned}
$$

where $\Phi_{p}$ and $\Phi_{f}$ are the pore and fracture porosities, respectively; $D_{i j k l}$ and $\Gamma_{i j} \neq \Pi_{i j}$ and $b$ are effective elastic coefficients, $K_{f i j}$ is the acoustic permeability of the fracture system, and $p$ is the pressure in the pores and fractures. Model $B-A$ is a Biot's model [see equations (1)-(4)]. However, in contrast with the original Biot's model, we can prove that the symmetry between $\Gamma_{i j}$ and $\Pi_{i j}$ is broken: $\Gamma_{i j} \neq \Pi_{i j}$. The quality factor is $Q^{-1}=O\left(Q_{f}^{-1}\right)=O(1)$.

\section{Model B-B: Equal separations of scales}

Model $B-B$ is characterized by $\ell / \ell^{\prime}=O\left(\ell^{\prime} / \lambda\right)$. This dualporosity model shows strong interactions between pore flow and fracture flow. The regime of the porous matrix is a Biot's regime of model $B$ and gives rise to squirt flow. Attenuation is from both pore flow and fracture flow: $Q^{-1}=O\left(Q_{f}^{-1}+Q_{p}^{-1}\right)=O(1)$. Homogenization yields a model similar to model $B-A$. However, model $B-B$ has important new features. First, the effective coefficients $D_{i j k l}, \Gamma_{i j} \neq \Pi_{i j}$ and $b$ are now complex valued and frequency dependent. At the macroscopic scale, the saturated microporous matrix is seen as a porous medium that undergoes a time-dependent consolidation process. Second, $p$ is the pressure in the fracture only: $p=p_{f}, p_{p} \neq p_{f}$. Note that the symmetry remains broken: $\Gamma_{i j} \neq \Pi_{i j}$.

\section{Model $B-C$ : High pore-fracture separation of scales}

In this case the scale ratios are such that $\ell / \ell^{\prime}=O\left(\left(\ell^{\prime} / \lambda\right)^{2}\right)$. The microporous system behaves like a single-phase elastic medium (model $C$ ). As a consequence, attenuation is obtained from the fracture system only: $Q^{-1}=O\left(Q_{f}^{-1}\right)=O(1)$. Again, the homogenized model is similar to equations (16)-(19). As in model $B-A, D_{i j k l}, \Gamma_{i j}, \Pi_{i j}$ and $b$ are real-valued effective elastic coefficients, but they differ from those in model $B-A$. As in model $B-B$, we have $p=p_{f}, p_{p} \neq p_{f}$. Note, however, that the symmetry $\Gamma_{i j}=\Pi_{i j}$ is now verified. In this respect, model $B-C$ is similar to a Biot's model.

\section{Model $B-D$ : Strongly high pore-fracture separation of scales}

We consider now the case $\ell / \ell^{\prime}=O\left(\left(\ell^{\prime} / \ell^{\prime \prime}\right)^{n}\right), n>4$. The microporous system behaves like a single-phase viscoelastic medium (model $D$ ). However, viscoelastic effects are quite low. Attenuation is given by $Q^{-1}=O\left(Q_{f}^{-1}\right)=O(1)$.

The upscaled model is again described by equations (16)(19), in which $D_{i j k l}, \Gamma_{i j}=\Pi_{i j}$, and $b$ are effective viscoelastic coefficients, but they differ from those in model $B-B$. As in models $B-B$ and $B-C$ we have $p=p_{f}, p_{p} \neq p_{f}$. The symmetry $\Gamma_{i j}=\Pi_{i j}$ is verified. Model $B-D$ is similar to a Biot's model with a viscoelastic porous matrix.

For a precise interpretation of the models, we define the following times,

$$
\tau_{p}=\frac{\ell^{\prime 2}}{c_{p} K_{p}}, \quad \tau_{f}=\frac{\lambda^{2}}{d_{f} K_{f}},
$$

that characterize the physical processes in the porous matrix and the fracture network, respectively, where $c_{p}$ and $d_{f}$ are the bulk moduli of the pore and the fracture frames and where $K_{p}$ and $K_{f}$ are the permeabilities of both porous systems. We have $c_{p}=O\left(d_{f}\right)$ and, in the viscous regime, $K_{p}=O\left(\ell^{2} / \mu\right), K_{f}=O\left(\ell^{\prime 2} / \mu\right)$. Analysis of the order of magnitude of their ratio,

$$
\frac{\tau_{p}}{\tau_{f}}=O\left(\frac{\ell^{\prime 4}}{\ell^{2} \lambda^{2}}\right)=O\left(\alpha^{-2} \beta^{2}\right),
$$

which depends only on the scale ratios, provides information on the intensity of fluid exchange between both porous systems with respect to fracture flow. It also indicates whether the porous matrix may be subject to consolidation.

Model B-A is characterized by $\tau_{p}=O\left(\beta \tau_{f}\right) \ll \tau_{f}$, which indicates that fluid exchange between both porous structures is instantaneous whereas fracture flow occurs later. Furthermore, the microporous matrix consolidates at $\tau_{p}$. In model B-B we get $\tau_{p}=O\left(\tau_{f}\right)$, which gives rise to strong interactions between pore flow and fracture flow. Finally, for models B-C and B-D the characteristic times are such that $\tau_{p} \gg \tau_{f}$. As a result, fluid exchange is negligible a time $\tau_{f}$ with respect to fracture flow, and the microporous matrix does not consolidate at $\tau_{f}$.

The domains of validity of models $B-A, B-B, B-C$, and $B-D$, which are defined by means of the relative orders of magnitude of the scale ratios $\alpha$ and $\beta$ [equation (15)], are shown in Figure 3 . 


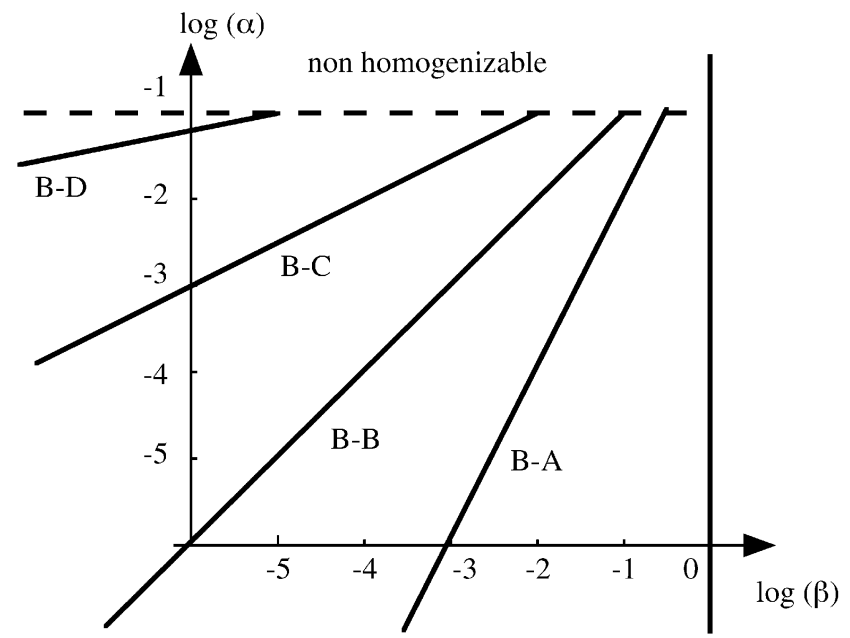

FIG. 3. Domains of validity of the four dual-porosity models.

\section{CONCLUSION}

An important conclusion drawn from this study is that there exist four distinct macroscopic two-phase models for describing seismic wave propagation in fractured porous media. The choice of model is conditioned by the relative order of magnitude of the scale ratios. In a given medium, $\ell$ and $\ell^{\prime}$ are related to the knowledge of the structure of the material and are therefore defined, whereas $\lambda$ depends on the excitation. Therefore, the choice of the macroscopic model is fully conditioned by the excitation of the medium.

Our presentation is restricted to cases of interest for geophysical applications, i.e., two-phase behaviors for which the signature of fluid flow is contained in the model. These results can easily be extended to the three other possible behaviors for the fractured system. The analysis is also limited to the case of a viscous regime defined by $K_{p}=O\left(\ell^{2} / \mu\right), K_{f}=O\left(\ell^{\prime 2} / \mu\right)$, which corresponds to the range of frequencies of seismic waves. Within the inertial regime, we have $K_{p}=O\left(1 / i \omega \rho_{f}\right)=O\left(K_{f}\right)$, which gives rise to different behaviors.

The four distinct two-phase, dual-porosity behaviors are shown in Figure 3 with respect to the orders of magnitude of the scale ratios. For $\log \left(\ell / \ell^{\prime}\right)=1$, the dual-porosity medium has a single-porosity behavior. In the case under consideration here, we retrieve Biot's model. For $\log \left(\ell^{\prime} / \ell^{\prime \prime}\right)>-1$, i.e., $\ell^{\prime} / \ell^{\prime \prime}>0.1$, the separation of scales of the fractured porous medium becomes quite poor. This bound is generally considered a rea- sonable limit for the existence of a macroscopic equivalent behavior.

Biot's theory and our results are valid for an incompressible or a slightly compressible fluid. For all models we present, Gassman's limit is reached when $\omega \rightarrow 0$. For strongly compressible fluids, Biot's model would be modified (the macroscopic mass balance is changed) and Gassman's limit would not be reached.

\section{ACKNOWLEDGMENTS}

J.-L. A. thanks Mobil Oil Technology Company for financial support from a research gift. Serge Shapiro, Boris Gurevich, and two other reviewers are greatly acknowledged for useful comments that helped improve the final manuscript.

\section{REFERENCES}

Auriault, J.-L., 1980, Dynamic behavior of a porous medium saturated by a Newtonian fluid: Internat. J. Eng. Sci., 18, 775-785.

1991a, Dynamic behaviour of porous materials, in Bear, J. and Corapcioglu, M. Y., Eds., Transport processes in porous media: Kluwer, 471-519.

1991b, Heterogeneous medium: Is an equivalent description possible?: Internat. J. Eng. Sci., 29, 785-795.

Auriault, J.L., and Boutin, C., 1992, Deformable porous media with double porosity, quasi-statics, I: Coupling effects: Trans. in Porous Media, 7, 63-82.

1993, Deformable porous media with double porosity, quasistatics, I: Memory effects: Trans. in Porous Media, 10, 153-169.

- 1994, Deformable porous media with double porosity, III: Acoustics: Trans. in Porous Media, 14, 143-162.

Barenblatt, G. I., Zheltov, Y. P., and Kochina, I. N., 1960, Basic concepts in the theory of seepage of homogeneous liquids in fissured rocks: Prikladnaya Matematika i Mekhanika, 24, 852-864.

Bensoussan, A., Lions, J. L., and Papanicolaou, G., 1978, Asymptotic analysis for periodic structures: North Holland Publ. Co.

Berryman, J. G., and Wang, H. F., 2000, Elastic wave propagation and attenuation in a double-porosity medium dual-permeability medium: Internat. J. Rock Mech. Min. Sci., 37, 63-78.

Beskos, D. E., 1989, Dynamics of saturated rocks, I: Equations of motion: J. Eng. Mech., Am. Soc. Civ. Eng., 115, 983-995.

Biot, M. A., 1956, The theory of propagation of elastic waves in a fluid saturated porous solid, I-Low frequency range; II-Higher frequency range: J. Acoust. Soc. Am., 28, 168-191.

Burridge, R., and Keller, J. B., 1981, Poroelasticity equations derived from microstructure: J. Acoust. Soc. Am., 70, 1140-1146.

Keller, J. B., 1977, Effective behaviour of heterogeneous media, in Landman, U., Ed., Statistical mechanics and statistical methods in theory and application: Plenum Press, 631-644.

Levy, T., 1979, Propagation of waves in a fluid saturated porous elastic solid: Internat. J. Eng. Sci., 17, 1005-1014.

Sanchez-Palencia, E., 1980, Non-homogeneous media and vibration theory: Lecture notes in physics series, 127, Springer-Verlag Berlin.

Wilson, R. K., and Aifantis, E. C., 1984, A double porosity model for acoustic wave propagation in fractured-porous rocks: Internat. J. Eng. Sci., 22, 1209-1217. 